\title{
Peuplements malacologiques lacustres en relation avec la physico- chimie de l'eau et des sédiments II. Les espèces
}

\author{
J. Mouthon 1
}

Mots clés : Mollusques, lacs, descripteurs physico-chimiques, analyse multivariée.

L'application d'une analyse multivariée (AFC) à 4 descripteurs synthétiques de la colonne d'eau et du sédiment nous a fourni une distribution dans le plan factoriel, des 18 lacs de notre échantillon. A partir de celle-ci, 4 types de milieux ont pu être définis.

La projection, en éléments supplémentaires dans le plan de l'analyse des variables du milieu, des effectifs de 26 espèces de mollusques a permis d'étudier les concordances entre types de systèmes et profils des espèces à 4 classes de profondeur : $0-1,3-5,10-15$ et $\geqslant 20 \mathrm{~m}$.

Affinité et tolèrance particulières des espèces pour chaque type de lac sont définis.

A l'échelle d'un écosystème lacustre, on observe que ses capacités d'oxygénation et la teneur en matière organique de ses sédiments jouent un rôle essentiel dans la distribution des espèces, celles-ci présentant une sensibilité différentielie vis-à-vis de ces deux facteurs, en fonction de la profondeur.

Snail and bivalve populations analysed in relation to physico-chemical quality of lakes in Eastern France. II. The species. Keywords : Mollusca, lakes, physico-chemical descriptors, multivariate analysis.

The application of Correspondence Analysis to synthetic descriptors of the water column and sediment gave an ordination, in the factorial plane, for the 18 lakes in our sample.

Based on this analysis, 4 types of lakes were defined.

The projection of abundances of 26 species as supplementary variables on the plane F1F2 stressed the relationships between lake categories and species profiles at 4 depths : 0-1, 3-5, 10-15 and $\geqslant 20 \mathrm{~m}$.

Particular affinity and tolerance of species for each type of lake were defined.

Oxygen supply and organic matter content of sediments both play a major role in species distribution in a lake. The species studied have different ranges of sensitivity to these two parameters that vary according to depth.

\section{Introduction}

Dans un précédent travail (Mouthon 1990), l'importance des conditions climatiques dans la distribution des malacocénoses des écosystèmes lacustres européens a été observée. Cependant, à une dimension spatiale plus restreinte (échelle régionale), ce n'est plus la température qui jouerait le principal rôle discriminant, d'autres facteurs étant susceptibles d'intervenir.

1. CEMAGREF, 3 bis Quai Chauveau, 69336 Lyon Cedex 09 , France.
En Norvège, où les mollusques ont fait l'objet de recherches approfondies, la dureté des eaux essentiellement calcique, la présence de macrophytes, le $\mathrm{pH}$ et la nature du substrat expliquent l'essentiel de la distribution des Gastéropodes dans la zone littorale des systèmes lacustres (Ökland 1990).

Chez les Sphaeriidae, moins étudiés, la rudesse des climats arctiques, les trop basses valeurs du $\mathrm{pH}$ et de la concentration des eaux en calcium, les fortes teneurs des eaux en acides humiques sont considérées comme des facteurs limitant la distribution de ces Bivalves (Meier-Brook 1963, Aho 1966, Ökland \& Kuiper 1982). 
Une première confrontation des peuplements malacologiques, au moyen de critères globaux, à la distribution, dans le plan factoriel F1F2, de 18 systèmes lacustres (Mouthon, sous presse) a montré :

- le caractère d'euryécie générale des mollusques et le rôle plus déterminant des sels de calcium sur la densité des peuplements que sur la richesse spécifique ;

- l'importance de l'oxygène dissous et de la matière organique des sédiments dans la distribution des malacocénoses lacustres ;

- l'existence d'exigences écologiques propres aux deux classes de mollusques : Gastéropodes et Bivalves, en fonction de la profondeur considérée ;

- que le mode de fonctionnement des lacs, défini à partir des conditions régnant dans leur zone profonde, détermine non seulement la composition des peuplements profonds mais également celle des malcocénoses littorales.

Dans la seconde partie, nous nous proposons d'analyser la projection dans le plan de l'analyse des variables du milieu des effectifs des 26 espèces les plus fréquentes. A partir de leur position par rapport aux 4 types de systèmes préalablement définis, nous tenterons de préciser leur préférendum et de suivre l'évolution de leurs exigences écologiques en fonction de la profondeur.

Situés dans l'est de la France, les 18 milieux faisant l'objet de ces recherches sont répertoriés dans le tableau 1. Parmi ceux-ci, seuls les lacs vosgiens de Longemer et Gérardmer possèdent un bassin versant crystallophyllien, les autres plans d'eau appartiennent au massif jurassien ou à la zone alpine dont le substrat est essentiellement calcique.

\section{Matériel et méthodes}

Les données physico-chimiques utilisées dans ce travail ont été recueillies par différents organismes tels que les Services Régionaux de l'Aménagement des Eaux de Franche-Comté et de Rhône-Alpes, le Centre d'étude des eaux continentales de l'Université de Franche-Comté, le CEMAGREF et figurent dans une vingtaine de rapports que l'on peutconsulter auprès de ces services.

L'analyse des sédiments et de l'eau interstitielle a été conduite suivant un protocole décrit par Rofes (1980), puis par des travaux du Centre d'Etude des Eaux Continentales de l'Université de FrancheComté (Verneaux et al. 1987).

Tableau 1. Répertoire des lacs étudiés.

Table 1. List of lakes in this study.

\begin{tabular}{lcccc}
\hline \multicolumn{1}{c}{ Lacs } & $\begin{array}{c}\text { Situation } \\
\text { géographique }\end{array}$ & $\begin{array}{c}\text { Altitude } \\
(\mathbf{m})\end{array}$ & $\begin{array}{c}\text { Superficie } \\
(\text { ha) }\end{array}$ & $\begin{array}{c}\text { Profondeur maximum } \\
(\mathbf{m})\end{array}$ \\
\hline Gérardmer & Vosges & 660 & 115,5 & 36 \\
Longemer & - & 750 & 76,2 & 30 \\
Nantua & Jura & 478 & 141 & 43 \\
Chalain & - & 500 & 231,8 & 30 \\
Grand Clairvaux & - & 534 & 63,46 & 20 \\
Sylans & - & 584 & $\mathbf{4 9 , 7 0}$ & 18 \\
Narlay & - & $\mathbf{7 5 0}$ & $\mathbf{4 1 , 6}$ & $\mathbf{4 0}$ \\
llay & - & $\mathbf{7 7 8}$ & $\mathbf{7 2 , 6}$ & $\mathbf{3 0}$ \\
Petit Maclu & - & $\mathbf{7 7 9}$ & $\mathbf{6 , 4}$ & $\mathbf{1 0}$ \\
Grand Maclu & - & $\mathbf{7 7 9}$ & $\mathbf{2 4 , 4}$ & $\mathbf{2 6}$ \\
Bonlieu & - & 803 & 17,36 & 16 \\
Saint Point & - & 849 & $\mathbf{3 9 8 , 2}$ & $\mathbf{4 0}$ \\
Rémoray & - & $\mathbf{8 5 0}$ & $\mathbf{9 5 , 3}$ & $\mathbf{2 5}$ \\
Abbaye & - & 879 & 92,45 & 19 \\
Les Rousses & - & 1059 & 89,8 & 15 \\
Laffrey & Alpes & 911 & 126,9 & 39 \\
Petitchet & - & 930 & 86 & 19 \\
Pierre-Chatel & - & 934 & 100,31 & 10 \\
\hline
\end{tabular}


Entre 0 et $-1 \mathrm{~m}$, les mollusques ont été prélevés à l'aide d'un troubleau à base rectangulaire $(25 \times$ $18 \mathrm{~cm}$ ). L'échantillonnage réalisé sur toute la largeur de la zone littorale représente une surface totale de 1, 2, parfois $3 \mathrm{~m}^{2}$ par point de prélèvement : leurs nombres varient de 3 à 13 en fonction de la superficie et de la morphométrie du milieu mais également de la diversité des habitats littoraux.

Les mollusques des zones profondes ont été récoltés à l'aide d'une benne de Friedinger ( $\mathrm{S}=350 \mathrm{~cm}^{2}$ ), trois prélèvements étant effectués à chaque point d'échantillonnage. Ceux-ci sont répartis sur deux, trois ou quatre transects, choisis en fonction des caractéristiques morphologiques des plans d'eau, à $3,5,10,15,20 \mathrm{~m}$, etc., jusqu'au point le plus profond de chaque cuvette.

Seuls les effectifs moyens par profondeur ou par niveau ont été retenus.

Afin de pouvoir comparer peuplements littoraux et profonds, la densité des espèces est, à chaque niveau d'échantillonnage, rapportée au $\mathrm{m}^{2}$.

Pour chaque lac, deux campagnes de prélèvement ayant généralement été effectuées entre les mois de juin et septembre, ce sont les effectifs moyens qui ont été pris en considération dans cette étude.

Les échantillons ont été tamisés à $630 \mu \mathrm{m}$, puis triés et déterminés à la loupe binoculaire.

L'hétérogénéité des donnóes disponibles, et surtout le fait que les échantillons physico-chimiques et biologiques ne proviennent pas des mêmes prélèvements, nous ont conduit à abandonner les valeurs paramétriques brutes au profit de critères synthétiques permettant de caractériser tes lacs de façon plus globale.

Deux catégories de variables ont été utilisées dans nos analyses:

1) Les unes, à caractère synthétique, sont des descripteurs physico-chimiques du milieu dont le choix a été discuté dans la première partie de ce travail (Mouthon, sous presse) ; seules les valeurs mesurées dans la zone profonde en fin de stratification estivale, l'une des deux périodes critiques pour ces lacs dimictiques, ont été retenues.

Il s'agit, respectivement pour l'eau et le sédiment, des rapports :

- concentration maximale en azote nitrique sur concentration maximale en sels ammoniacaux au point le plus profond, soit $\mathrm{NO}_{3} / \mathrm{NH}_{4}$ ou taux de nitrification généralement utilisé dans la littérature
(Hutchinson 1957, Wetzel 1975, Hakanson \& Jansson 1983).

- hauteur d'eau désoxygéné (avec $0_{2}<2 \mathrm{mg} / \mathrm{l}$ ) sur la profondeur maximale ou DEOX : coefficient de désoxygénation.

- carbonates de calcium sur perte au feu : $\mathrm{CaCO}_{3} / \mathrm{PF}$ ou sur carbone organique fortement corrélé avec PF (Mackereth 1966, Digerfeldt 1972).

- Azote Kjeldahl, également fortement corrélé à la perte au feu (Mackereth 1966, Kemp 1971, Verneaux et al. 1987) sur phosphore total : NKJ/PHOT.

2) Les autres variables représentent les effectifs moyens des différentes espèces au niveau de 4 classes de profondeur : dans la zone littorale entre 0 et $1 \mathrm{~m}$, puis à $3-5 \mathrm{~m}$, dans la zone profonde à $10-15 \mathrm{~m}$ et à partir de $20 \mathrm{~m}$.

Ces variables biologiques sont codées au moyen de 2 lettres (Tableau 2), la première représentant l'initiale du genre, la suivante caractérisant l'espèce.

Tableau 2. Liste et code des espèces utilisées dans l'analyse. Table 2. List and code of the species used in the analysis.

\begin{tabular}{ll}
\hline Code & Especes \\
\hline VC & Valvata cristata Müller \\
VP & Valvata piscinalis (Müller) \\
BT & Bithynia tentaculata (L.) \\
LS & Lymnaea stagnalis (L.) \\
LP & Lymnaea palustris (Müller) \\
LT & Lymnaea truncatula (Müller) \\
LA & Lymnaea auricularia (L.) \\
LE & Lymnaea peregra (Müller) \\
AS & Anisus spirorbis (L.) \\
GA & Gyraulus albus (Müller) \\
AC & Armiger crista (L.) \\
HC & Hippeutis complanata (L.) \\
PC & Planorbis carinatus (Müller) \\
AL & Acroloxus lacustris (L.) \\
AY & Anodonta cygnea (L.) \\
PA & Pisidium casertanum (Poli) \\
PO & Pisidium conventus Clessin \\
PH & Pisidium hibernicum Westerlund \\
PL & Pisidium lifleborgii Clessin \\
PM & Pisidium milium Held \\
PN & Pisidium nitidum Jenyns \\
PB & Pisidium obtusale (Lamarck) \\
PP & Pisidium personatum Malm \\
PS & Pisidium subtruncatum Malm \\
PT & Pisidium tenuilineatum Stelfox \\
SC & Sphaerium corneum (L.) \\
\hline &
\end{tabular}


L'hétérogénéité des valeurs des différents descripteurs physico-chimiques et biologiques nécessitant leur normalisation, les données brutes ont été transformées en classes. Les valeurs moyennes et maximales des effectifs moyens de chaque espèce par lac, sont considérées respectivement comme repère de la classe médiane et de la classe 5 (Verneaux 1973, Mouthon 1980). Les espèces présentant des effectifs moyens et maximums proches ont été regrotlpées dans la même classe (Tableau 3).

La matrice ainsi obtenue a été traitée au moyen d'une analyse factorielle des correspondances, dont le logiciel est extrait de la bibliothèque ADDAD. Le distance entre individus et le critère d'agrégation utilisés dans cette analyse sont respectivement le $\chi^{2}$ et le moment centré d'ordre 2 . Les traitements ont été effectués sur un ordinateur de type VAX.

\section{Résultats et discussion}

\subsection{Organisation des milieux dans l'espace factoriel}

L'application de l'analyse factorielle des correspondances aux données recodées, nous a fourni une image de la distribution des variables physicochimiques et des milieux dans le plan des deux premiers axes, représentant $83,8 \%$ de l'inertie totale du nuage, respectivement $50,9 \%$ pour F1 et $32,9 \%$ pour F2 (Fig.1).

Les variables s'opposent deux à deux : le taux de nitrification $\mathrm{NO}_{3} / \mathrm{NH}_{4}$ au coefficient de désoxygénation de la masse d'eau (DEOX) sur l'axe 1 et le rapport $\mathrm{CaCO}_{3} / \mathrm{PF}$ à NKJ/PHOT sur le deuxième facteur (Tableau 4).

Tableau 3. Classes utilisées pour coder les variables physico-chimiques ( $n$ ) et les effectifs moyens des espèces aux différentes profondeurs. Les 4 types de classes ont été déterminés en tenant compte des valeurs moyennes et maximales des effectifs moyens de chaque espèce.

Table 3. Classes used to code the physico-chemical variables and the average numbers of species at different depths. The four types of classes have been determined by taking into account the mean and maximum values of the average numbers of each species.

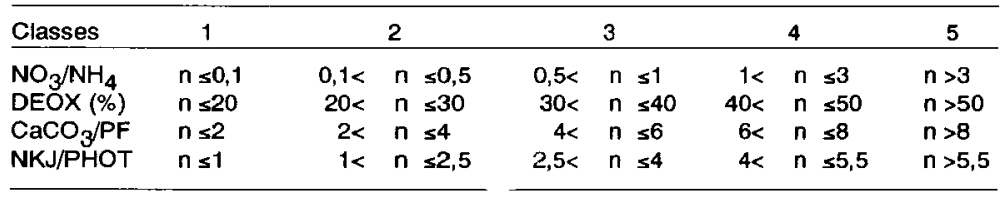

\begin{tabular}{|c|c|c|c|c|c|c|}
\hline Classes & 1 & 2 & 3 & 4 & 5 & Nom des especes \\
\hline \multicolumn{7}{|l|}{ Types } \\
\hline 1 & $1-2$ & $3-4$ & $5-6$ & $7-10$ & $>10$ & $\begin{array}{l}\text { Valvata cristata, Lymnaea stagnalis, } \\
\text { Lymnaea peregra, Lymnaea truncatula, } \\
\text { Lymnaea auricularia, Lymnaea palustris, } \\
\text { Anisus spirorbis, Acroloxus lacustris, } \\
\text { Anodonta cygnea, Sphaerium comeum, }\end{array}$ \\
\hline 2 & $1-2$ & $3-7$ & 8-15 & $16-30$ & $>30$ & $\begin{array}{l}\text { Gyraulus albus, Hippeutis complanata, } \\
\text { Pisidium tenuilineatum }\end{array}$ \\
\hline 3 & $1-5$ & $6-15$ & $16-30$ & $31-60$ & $>60$ & $\begin{array}{l}\text { Valvata piscinalis, Bithynia tentaculata, } \\
\text { Armiger crista, Planorbis carinatus, } \\
\text { Pisidium casertanum, Pisidium conventus, } \\
\text { Pisidium lilleborgii, Pisidium milium, } \\
\text { Pisidium obtusale, Pisidium personatum }\end{array}$ \\
\hline 4 & $1-20$ & $21-50$ & $51-100$ & $101-200$ & $>200$ & $\begin{array}{l}\text { Pisidium hibernicum, Pisidium nitidum, } \\
\text { Pisidium subtruncatum }\end{array}$ \\
\hline
\end{tabular}




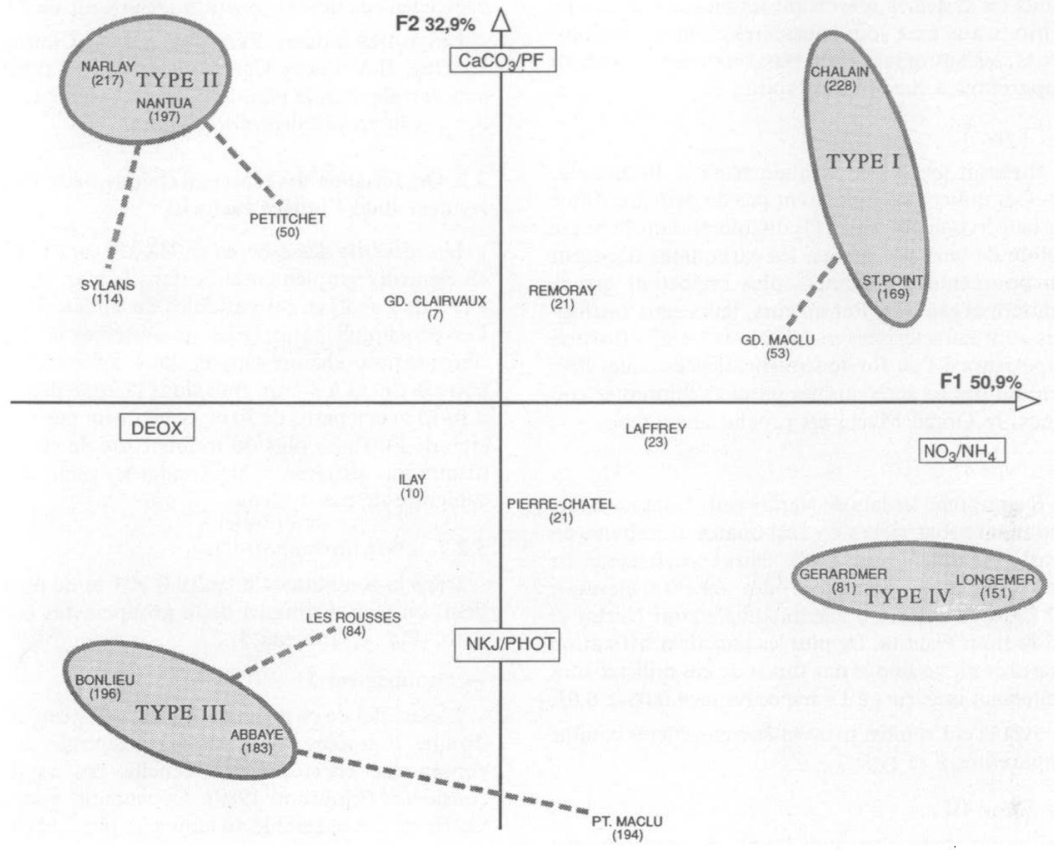

Fig. 1. Distribution des lacs et des variables physico-chimiques dans le plan F1F2. Entre parenthèses : somme des contributions aux axes 1-2 des milieux.

Fig. I. Distribution of the lakes and the physico-chemical variables in the plane F1F2. In parentheses: sum of the contributions in axes 1-2 of the lakes.

Tableau 4. Qualité de la représentation (COR) et contribution relative des variables physico-chimiques à l'inertie des axes 1 et 2 (CTR). Table 4. Quality of the projection (COR) and the relative contribution of the physico-chemical variables to the inertia of axes 1 and 2 (CTR).

\begin{tabular}{lrrrrrr}
\hline $\mathrm{J}$ & axe 1 & COR & CTR & axe 2 & COR & CTR \\
\hline $\mathrm{NO}_{3} / \mathrm{NH}_{4}$ & 517 & 873 & 561 & -69 & 16 & 14 \\
$\mathrm{DEOX}_{\mathrm{CaCO}} / \mathrm{PF}$ & -425 & 799 & 437 & -42 & 8 & 6 \\
$\mathrm{NKJ} / \mathrm{PHOT}$ & 9 & 0 & 0 & 443 & 923 & 646 \\
& -29 & 6 & 2 & -300 & 629 & 333 \\
\hline
\end{tabular}


Quatre grands types de milieux ont été définis. Seuls les systèmes présentant les plus fortes contributions aux axes sont considérés comme représentatifs, les autres lacs étant plus ou moins fortement apparentés à chacun des types.

\section{- Type I}

Il réunit les lacs de Chalain et de St Point (Fig. 1). Ces milieux ne présentent pas de déficit critique de leur hypolimmion en $\mathrm{O}_{2}$ dissous et dans la phase solide de leurs sédiments, les carbonates occupent un pourcentage nettement plus important que la matière organique. Par ailleurs, leurs eaux profondes sont caractérisées par des taux de nitrification supérieurs à 1 en fin de stratification estivale. Présentant des caractéristiques physico-chimiques voisines, le Grand Maclu est proche de ce type.

\section{- Type II}

Il comprend les lacs de Narlay et de Nantua. Leurs sédiments sont riches en carbonates et pauvres en matières organiques mais leurs coefficients de désoxygénation atteignent des valeurs élevées: $57 \%$ de la hauteur d'eau maximale pour Narlay et $75 \%$ pour Nantua. De plus les taux de nitrification mesurés au voisinage des fonds de ces milieux sont nettement inférieurs à 1 : respectivement 0,08 et 0,05 .

Sylans et Petitchet peuvent être considérés comme apparentés à ce type.

\section{- Type III}

Les lacs de Bonlieu et de l'Abbaye appartiennent à ce type. Ils sont caractérisés par des sédiments riches en matières organiques (carbones organiques et azote total) et des coefficients de désoxygénation élevés, respectivement 53 et $51 \%$ de la hauteur d'eau maximale. Par ailleurs, le taux de nitrification de leurs eaux profondes demeure faible : 0,02 pour Bonlieu, 0,2 pour l'Abbaye. Ces milieux présentent une forte tendance à la dystrophie.

Les lacs des Rousses et du Petit-Maclu, ce dernier ne présentant pas de période hypoxique en raison de sa faible profondeur $(-10 \mathrm{~m})$, sont proches de ce type.

\section{- Type IV}

Il comprend essentiellement les lacs vosgiens de Longemer et Gérardmer, à bassin versant crystallophyllien et donc pauvres en sels de calcium. Seul le dernier présente un déficit en oxygène dissous de son hypolimnion en fin de stratification estivale, son coefficient de désoxygénation DEOX est de $29 \%$.

Les autres milieux Rémoray, Grand Clairvaux, Laffrey, Ilay, Pierre-Chatel occupent une position subcentrale dans le plan F1F2 et représentent donc des cas intermédiaires non typiques.

\subsection{Organisation des espèces à chaque classe de pro- fondeur dans l'espace factoriel}

Les effectifs des espèces en classes sont projetés en éléments supplémentaires dans le plan factoriel F1F2 de l'analyse des variables du milieu (Fig. 2). Les proximités entre types de systemes et profils obtenus pour chaque espèce, dans la zone littorale entre $0-1 \mathrm{~m}$ et à $3-5 \mathrm{~m}$, puis dans la zone profonde à $10-15 \mathrm{~m}$ et à partir de $20 \mathrm{~m}$, constituent une expression de l'affinité plus ou moins forte de ces organismes aux différentes profondeurs, pour chaque catégorie de plans d'eau.

\subsubsection{Zone littorale $(0-1 \mathrm{~m})$}

Dans la zone littorale, entre 0 et $1 \mathrm{~m}$ de profondeur, on peut distinguer deux groupements principaux (Fig. 2, Tableau 5).

\section{- Groupement 1}

L'essentiel de ce groupement est constitué d'une dizaine d'espèces à position subcentrale et par conséquent eurytopes à l'échelle des systèmes considérés (Mouthon 1989). Cependant, à la périphérie de cet ensemble se trouvent plusieurs espèces dont les préférendums apparaissent plus nettement.

Parmi celles-ci, Bithynia tentaculata et Anodonta cygnea qui occupent une position intermédiaire entre les lacs des types I et II, apparaissent plutôt tolérantes aux conditions régnant dans les lacs hypoxiques. En revanche, d'autres espèces comme Pisidium milium, $P$. hibernicum et Lymnaea stagnalis sont plus directement associées aux lacs du type I, ceux du type IV n'hébergeant que des malacocénoses très réduites dans leur zone littorale.

\section{- Groupement 2}

Il regroupe neuf espèces plus ou moins fortement associées aux lacs riches en matière organique du type III. Parmi ces mollusques on compte 8 Gastéropodes dont 4 Planorbidae : Anisus spirorbis, Hippeutis complanata, Armiger crista et Gyraulus albus et un Bivalve : Pisidium obtusale. 


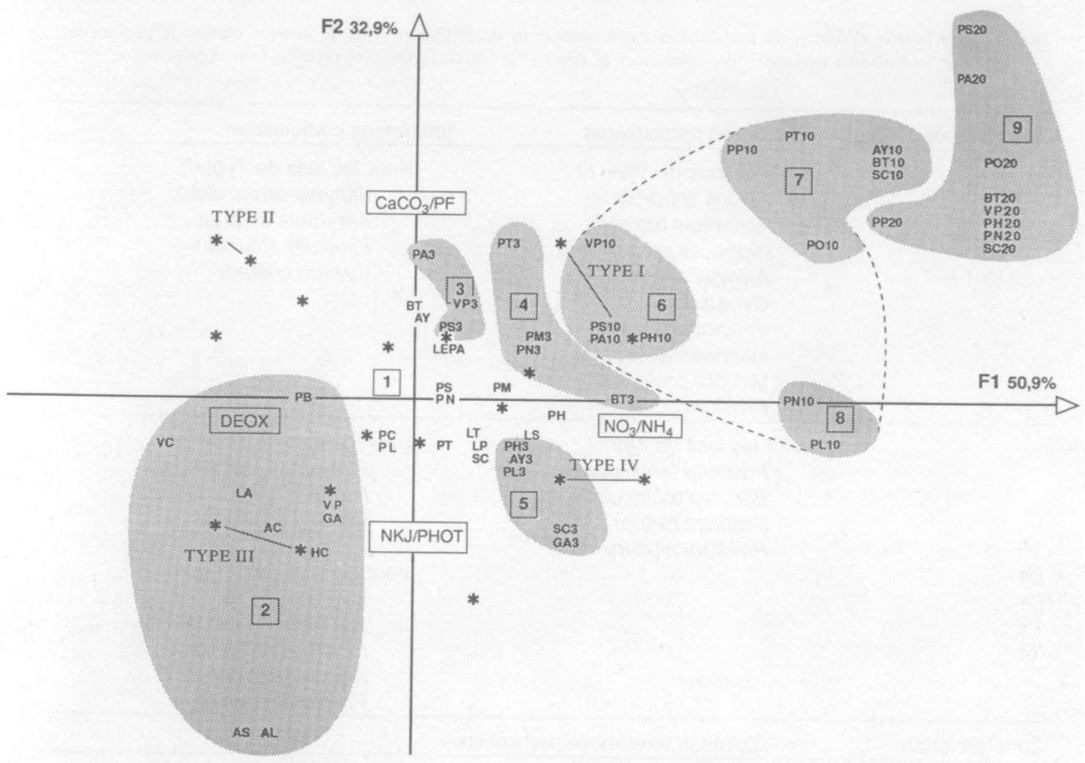

Fig. 2. Projection en éléments supplémentaires des classes d'effectifs des espèces dans le plan F1F2.

: position de chaque lac.

La position de chaque espèce aux profondeurs $0-1,3-5,10-15$ et $\geqslant 20 \mathrm{~m}$ est indiquée par son code seul ou suivi des chiffres $3,10,20$. Pour la signification des codes voir Tableau 2.

Fig. 2. Projection as supplementary variables of the species abundance classes in the plane F1F2.

* : position of each lake.

The position of each species at depths of $0-1,3-5,10-15$ and $\geqslant 20 \mathrm{~m}$ is indicated by only its code or followed by letters $3,10,20$. See Table 2 for the meaning of the codes.

Toutes les espèces appartenant à ce groupement et plus particulièreent Anisus spirorbis et Acroloxus lacustris, étroitement associées aux systèmes du type III, peuvent être qualifiées de saprobiontes.

\subsubsection{Zone littorale $(3-5 \mathrm{~m})$}

En fonction de leur affinité pour les différents types de lacs, les espèces présentes entre 3 et $5 \mathrm{~m}$ de profondeur, constituent trois groupements.

\section{- Groupement 3}

Il réunit trois espèces, les Bivalves Pisidium casertanum et $\boldsymbol{P}$. subtruncatum et le Gastéropode $\mathrm{Val}$ vata piscinalis qui occupent une position intermédiaire entre les lacs des types I et 1I. Ce groupement est plus proche des lacs hypoxiques que les deux suivants. Ceci montre que ces espèces sont surtout tolérantes aux conditions régnant dans les lacs du type II. 
Tableau 5. Affinités et tolérances particulières des espèces pour les différents types de lacs à 4 classes de profondeur. Table 5. Affinities and particular tolerances of species for the different lake types at four depth-classes.

\begin{tabular}{|c|c|c|c|}
\hline Zone littorale & affinités particulières & & tolérances particulières \\
\hline $0-1 \mathrm{~m}$ & $\begin{array}{c}\text { pour les lacs de Type III } \\
\text { Anisus spirorbis } \\
\text { Acroloxus lacustris } \\
\text { Hippeutis complanata } \\
\text { Armiger crista } \\
\text { Gyraulus albus } \\
\text { Valvata piscinalis } \\
\text { Lymnaea auricularia } \\
\text { Valvata cristata } \\
\text { Pisidium obtusale }\end{array}$ & & $\begin{array}{c}\text { Pour les lacs de Type II } \\
\text { Bithynia tentaculata } \\
\text { Anodonta cygnea } \\
\text { Pisidium abtusale } \\
\text { Valvata cristata }\end{array}$ \\
\hline 3-5 m & $\begin{array}{l}\text { pour les lacs de Type I } \\
\text { Pisidium tenuilineatum } \\
\text { Bithynia tentaculata } \\
\text { Pisidium milium } \\
\text { Pisidium nitidum }\end{array}$ & . & $\begin{array}{l}\text { pour les lacs de Types II } \\
\text { Pisidium casertanum } \\
\text { Pisidium subtruncatum } \\
\text { Valvata piscinalis } \\
\\
\text { pour les lacs de Type III } \\
\text { Gyraulus albus } \\
\text { Sphaerium corneum } \\
\text { Pisidium lilljeborgii } \\
\text { Anodonta cygnea } \\
\text { Pisidium hibernicum }\end{array}$ \\
\hline Zone profonde & \multicolumn{3}{|c|}{ Affinités et tolérances particulières } \\
\hline $10-15 \mathrm{~m}$ & $\begin{array}{l}\text { pour les lacs de Type I } \\
\text { Anodonta cygnea } \\
\text { Pisidium tenuilineatum } \\
\text { Sphaerium corneum } \\
\text { Bithynia tentaculata } \\
\text { Pisidium conventus } \\
\text { Pisidium personatum } \\
\text { Pisidium hibernicum } \\
\text { Valvata piscinalis } \\
\text { Pisidium casertanum } \\
\text { Pisidium subtruncatum } \\
\\
\text { pour les lacs des types I et IV } \\
\text { Pisidium nitidum } \\
\text { Pisidium filleborgii }\end{array}$ & ) & $\begin{array}{l}\text { espèces plus } \\
\text { tolérantes }\end{array}$ \\
\hline $220 \mathrm{~m}$ & $\begin{array}{l}\text { pour les lacs de Type I } \\
\text { Pisidium subtruncatum } \\
\text { Pisidium casertanum } \\
\text { Pisidium conventus } \\
\text { Bithynia tentaculata } \\
\text { Valvata piscinalls } \\
\text { Pisidium hibernicum } \\
\text { Pisidium nitidum } \\
\text { Sphaerium corneum } \\
\text { Pisidium personatum }\end{array}$ & $\longrightarrow$ & espèce plus tolérante \\
\hline
\end{tabular}




\section{- Groupement 4}

Associées aux lacs du type I et opposées à la fois aux systèmes des types III et II, les 4 especes de ce groupement présentent une certaine sensibilité aux conditions régnant dans ces deux derniers types de milieux. Elles sont plus saprophobes que les espèces des groupements 3 et 5 .

\section{- Groupement 5}

Ces 5 espèces occupent une position intermédiaire entre les lacs des types I et III, les milieux du type IV, très pauvres en mollusques à ces profondeurs, ayant peu d'influence sur leur profil dans cette analyse. Opposées aux lacs hypoxiques du type II, ces espèces apparaissent donc plus sensibles aux conditions régnant dans ces systèmes. Elles sont donc à la fois également plus oxyphiles que les Bivalves du groupement 3 et plus saprobiontes que les espèces des groupements 3 et 4 .

\subsubsection{Zone profonde $(10-15 \mathrm{~m})$}

Les trois groupements qui s'individualisent à ces profondeurs sont directement associés aux systèmes du type I.

Moins fortement opposées aux lacs des types II et III, les 4 espèces du groupement 6 sont donc plus tolérantes aux conditions régnant dans ces milieux que celles des noyaux 7 et 8 . Les deux pisidies appartenant à ce dernier groupement : Pisidium nitidum et $\boldsymbol{P}$. lilljeborgii présentent également une affinité avec les lacs du type IV, pauvres en sels de calcium ; elles sont donc moins calciphiles que leurs congénères.

\subsubsection{Zone profonde ( $\geqslant 20 \mathrm{~m}$ )}

Le groupement 9 renferme toutes les espèces qui atteignent la profondeur de $-20 \mathrm{~m}$; il est fortement associé aux lacs du type I.

\subsection{Discussion}

La classification des systèmes obtenue ici apparaît très voisine de celle proposée par Verneaux et al. (1987) pour 10 de ces lacs, à partir des rapports $\mathrm{CaCO}_{3} / \mathrm{PF}$ et $\mathrm{Ca} / \mathrm{Si}$ relatifs à la strate superficielle $(0-5 \mathrm{~cm})$ de leurs sédiments profonds.

Les Planorbidae, dont 4 espèces appartiennent au groupement 2 , sont connus pour leur affinité particulière pour les biotopes riches en végétation et en matière organique (Germain 1931, Boycott 1936, Adam $1960, \ldots)$. Toutefois, la position subcentrale de Planorbis carinatus montre que cette espèce eurytope est bien moins inféodée aux systèmes du type III que ses congénères.

Associées aux lacs du type III, les deux espèces de Valvatidae présentent néanmoins des exigences écologiçues fort différentes. Plus résistantes à la dessication (Yacine-Kassab 1979), Valvata cristata apparaî̀ bien moins exigente vis-à-vis des conditions d'oxygénation des systèmes que Valvata piscinalis.

Bien qu'il occupe une position intermédiaire entre les lacs de types II et III, Pisidium obtusale peut être rattache au groupement 2 . Electif des faciès riches en matières organiques, ce Bivalve capable de supporter des conditions extrêmes de pH et de température, contrairement à la plupart des autres pisidies (Favre 1927, Kuiper \& Wolff 1970, Meier-Brook 1975, Bleck \& Heitkamp 1980, Ökland \& Kuiper 1980), colonise aussi bien le littoral des lacs du type III que celui des systèmes hypoxiques du type II, à marnage plus ou moins important tels que Sylans et Petitchet.

Dans la zone littorale, entre 3 et $5 \mathrm{~m}$, les espèces du groupement 3 s'avèrent bien moins sensibles que celles des groupements 4 et 5 aux conditions régnant dans les lacs du type 2 où associés à ce type ; trois de ces systèmes : Nantua, Petitchet et Narlay sont connus pour avoir reçu des apports excessifs en nutriments durant plusieurs années (Vivier \& Serruya 1966, Gay 1976, Masson et al. 1978).

Déjà perceptible aux profondeurs de 10 et $15 \mathrm{~m}$, on observe une différence de sensibilité entre les deux Bivalves colonisant les zones profondes et abyssales des systèmes lacustres médio-européens : Pisidium conventus et $P$. personatum (Mouthon \& Kuiper 1987) ; ce dernier se révèle moins exigeant que son congénère.

Absente de la zone profonde des systèmes objets de cette étude, Lymnaea peregra est une espèce eurybathe, capable de coloniser la plaine abyssale de grands lacs comme le Léman, lorsque les conditions d'oxygénation n'y sont pas limitantes (Forel 1892-1904, Piaget 1913, Mouthon 1987). Cette limnée plus oxyphile dans la zone profonde que les espèces du groupement 9, peut donc également être considérée comme présentant une forte affinité avec les lacs du type I, caractérisés par des sédiments carbonatés et un hypolimnion sans déficit chronique en $\mathrm{O}_{2}$ dissous, ce qui était le cas du Léman avant les années 60 (Monod et al. 1984). 


\section{Conclusions}

A partir d'une première application de l'AFC à 4 variables synthétiques visant à caractériser d'une manière globale l'état de la masse d'eau et la composition de la phase solide du sédiment, 4 types de lacs ont pu être définis.

La projection en éléments supplémentaires du profil des espèces dans le plan F1F2 a permis de définir les relations existant entre mollusques et types de milieux. L'essentiel des résultats obtenus concernant affinité et tolérance particulière de ces espèces à chacune des profondeurs retenues : $0-1 \mathrm{~m}, 3-5 \mathrm{~m}$, $10-15 \mathrm{~m}$ et $\geqslant 20 \mathrm{~m}$ figurent dans le tableau 4 .

Par ailleurs, les exigences écologiques de plusieurs espèces littorales associées aux lacs de type III ont également été mises en évidence par l'analyse. Ainsi, Planorbis carinatus apparaît peu lié aux systèmes riches en matière organique contrairement aux autres Planorbidae, et parmi les Valvatidae : Valvata cristata est nettement moins oxyphile que $V$. piscinalis.

Des 4 types de milieux, seule la zone littorale des lacs du type III héberge des espèces présentant une affinité particulière pour ces systèmes riches en matière organique, les autres mollusques littoraux étant plutôt eurytopes à l'échelle des milieux considérés. En revanche, lorsque la profondeur augmente, à 3-5 m puis dans la zone profonde à $10-15 \mathrm{~m}$ et à partir de $20 \mathrm{~m}$, les espèces eurybathes sont de plus en plus fortement associées aux systèmes ne présentant pas de déficit critique de leur hypolimnion en $\mathrm{O}_{2}$ dissous et opposées aux lacs hypoxiques et (ou) présentant un excès de matière organique. Cette dernière exerce donc un rôle essentiel dans la distribution des mollusques en zone littorale. Dans la zone profonde, tant que les teneurs en matière organique accumulées dans les sédiments n'atteignent pas un seuil dépassant les capacités de minéralisation du système, c'est l'oxygène dissous qui demeure le principal facteur limitant. En revanche, dans la zone intermédiaire, aux profondeurs 3-5 $\mathrm{m}$, l'influence conjointe de ces deux facteurs s'observe sur les peuplements.

Après le facteur thermique, étroitement dépendant des conditions climatiques (Mouthon 1990), les capacités d'oxygénation d'un système et les teneurs en matière organique de ses sédiments, jouent donc un rôle essentiel dans la distribution des espèces qui présentent une sensibilité différentielle vis-à-vis de ces facteurs, en fonction de la profondeur.

\section{Remerciements}

L'auteur remercie vivement Monsieur J. Verneaux, Professeur à l'Université de Besançon, pour ses précieux conseils, ainsi que Madame Taillole qui a assuré la dactylographie du manuscrit.

Ce travail a été réalisé dans le cadre d'un programme financé par le SRETIE ( $\mathrm{N}^{\circ}$ 90207).

\section{Travaux cités}

Adam W. 1960. - Mollusques terrestres et dulcicoles. Faune de Belgique. Mollusques. 1. Institut royal des Sciences naturelles de Belgique Ed. Bruxelles, 402 p.

Aho J. 1966. - Ecological basis of the distribution of the lit toral freshwater molluscs in the vicinity of Tampere, South Finland. Ann. Zool. Fenn., $3:$ 287-322.

Bleck V. \& Heitkamp U. 1980. - Ökophysiologische Untersuchungen an Pisidium personatum Malm, 1855 und Pisidium obiusale (Lamarck, 1818) (Bivalvia, Sphaeridae). Zool. Anz., $205: 162-180$.

Boycott A.E. 1936. - The habitats of the freshwater Mollusca in Britain. J. anim. Ecol., $5: 116-186$.

Digerfeldt G. 1972. - The post-glacial development of Lake Trummen. Folia limnol, scand., $16: 1-104$.

Favre J. 1927. - Les mollusques post-glaciaires et actuels du bassin de Genève. Mém. Soc. Phys. Hist. nat. Genève, 40 : $171-434$

Forel F.A. 1892-1904. - Le Léman. Monographie limnologique, 3 vol., Slatkine Reprints, Genève (1969), 543, 651, 715 p.

Gay C. 1976 . - Contribution à l'étude écologique et à l'aménagement d'un lac de montagne : le lac de Petitchet (Isère). Thèse 3ème cycle, Univ. Grenoble, 124 p.

Germain L. 1931. - Faune de France. 22. Mollusques terrestres et fluviatiles. Librairie de la Faculté des Sciences Ed. Paris, 479-897.

Hakanson L. \& Jansson M. 1983. - Principle of Lake Sedimentology. Springer-Verlag Berlin, 316 p.

Hutchinson G.E. 1957. - A treatise on Limnology. 1. Geography, Physics, and Chemistry. John Wiley \& Sons Eds. New-York, 1015 p.

Kemp A.L.W. 1971. - Organic carbon and nitrogen in the surface sediments of lake Ontario. J. Sedim, Petrol, 41 : $337-548$.

Kuiper J.G.J. \& Wolff W.J. 1970. - The Mollusca of the estuarine region of the rivers Rhine, Meuse and Scheldt in relation to the hydrography of the area. III. The genus Pisidium. Basteria, $34: 1.42$.

Mackereth F.J.J. 1966. - Some chemical observations on postglacial lake sediments. Phil. Trans. r. Soc., Lond. 250: 167-213.

Masson J.P., Rouault J.V. \& Vidonne A. 1978. - Le lac de Narlay (Jura). Evolution de quelques paramètres physicochimiques en relation avec la présence d'Oscillatoria rubescens. In : Actes du colloque A.F.L. sur les lacs naturels, Chambéry. Univ. Savoie, CTGREF Ed. : 97-114.

Meier-Brook C. 1963. - Uber die Mollusken der Hochschwarzwald-und Hochvogesen Gewässer. Arch. Hydrobiol. Suppl, $28: 1-46$.

Meier-Brook C. 1975. - Der ökologische Indikatorwert mitteleuropäischer Pisidium- Arten (Mollusca, Eulamellibranchiata). Eiszeitalter u. Gegenwart, $26: 190-195$. 
Monod R., Blanc P. \& Corvi C. 1984. - Evolution physicochimique, 1. oxygène dissous, 2. les formes de l'azote. In : Le Leman, synthese 1957.1982. Commission internationale pour la protection des eaux du Léman contre la pollution. Lausanne : 89-120.

Mouthon J. 1980. - Contribution a l'écologie des Mollusques des eaux courantes. Esquisse biotypologique et donnés écologiques. Thèse $3^{e}$ cycle, Univ. Paris VI, $169 \mathrm{p}$.

Mouthon J. 1987. - Contribution à la connaissance des mollusques du Lac Léman. Intérêt de l'étude des malacocénoses pour apprécier la qualité biologique des sédiments de ce plan d'eau. Revue suisse Zool., 94 : 729-740.

Mouthon J. 1989. - Analyse de la distribution des malacocénoses de 23 lacs français. Ann/s Limnol, 25 : 205-213.

Mouthon J. 1990. - Importance des conditions climatiques dans la différenciation des peuplements malacologiques des lacs européens. Arch. Hydrobiol., $118: 353-370$.

Mouthon J. - Snail and bivalve populations analysed in relation to physico-chemical quality of lakes in Eastern France. I. General criteria for population analyses. Hydrobiologia, (in press).

Mouthon J. \& Kuiper J.G.J. 1987 - Inventaire des Sphaerijdae de France. 41. Secrétariat de la Faune et de la Flore et Mus. nat. Hist. nat. Eds. Paris, 60 p.

Ökland J. 1990. - Lakes and snails. Environment and Gastropoda in 1,500 Norwegian lakes, ponds and rivers. Universal Book Services/Dr. W. Backhuys, Oegstgeest, The Netherlands. $516 \mathrm{p}$.
Ökland K.A. 1971. - On the ecology of Sphaeriidae in a high mountain area in South Norway. Norw. J. Zool., 19 : 133-143.

Okland K.A. \& Kuiper J.G.J. 1980. - Smamuslinger (Sphaeriidae) i ferskvann i Norge - utbredelse, okologi og telasjon til forsuring (with summary in English). - SNSF - Project, Oslo-As, Norway, IR 61/80:85 p

Ökland K.A. \& Kuiper J.G.J. 1982. - Distribution of small mussels (Sphaeriidae) in Norway, with notes on their ecology. Malacologia, $22: 469-477$.

Piaget J.J. 1913. - Nouveaux dragages malacologiques dans la faune profonde du Léman. Zool. Anz., $42: 216-223$.

Rofes G. 1980. - Etude des sédiments. Méthodes de prélèvement et d'analyses pratiquées au laboratoire de sédimentologie. Etude $n^{\circ} 47$, CTGREF, Ministère de l'Agriculture : $50 \mathrm{p}$,

Verncaux J. 1973. - Cours d'eau de Franche-Comté (Massif du Jura) : recherches écologiques sur le réseau hydrographique du Doubs. Essai de biotypologie. These Doct., Fac. Sci. Univ. Besançon : 257 p.

Verneaux J., Remy F., Vidonne A. \& Guyard A. 1987. Caractères généraux des sédiments de 10 lacs jurassiens. Sci. Eau, $6: 107-128$.

Vivier P. \& Serruya S, 1966. - La pollution organique du lac de Nantua. Eou: 167-171.

Wetzel R.G. 1975. - Limnology. Saunders Ed. Philadelphia, $743 \mathrm{p}$.

Yacine-Kassab M. 1979. - Etude écologique des mollusques Gastéropodes d'eau douce de quelques milieux aquatiques peu profonds de la vallée du Rhône en amont de Lyon. Thèse Doct. Univ. Grenoble : 247 p. + annexes. 